\title{
Corrections
}

\section{Correction: Nyhuis et al., Evidence for the Integration of Stress-Related Signals by the Rostral Posterior Hypothalamic Nucleus in the Regulation of Acute and Repeated Stress-Evoked Hypothalamo-Pituitary- Adrenal Response in Rat}

In the article "Evidence for the Integration of Stress-Related Signals by the Rostral Posterior Hypothalamic Nucleus in the Regulation of Acute and Repeated Stress-Evoked Hypothalamo-Pituitary-Adrenal Response in Rat" by Tara J. Nyhuis, Cher V. Masini, Heidi E. W. Day, and Serge Campeau, which appeared on pages 795-805 of the January 20, 2016 issue, the authors regret the inadvertent omission of a study published by Kataoka et al. (2014) using some of the same approaches as those employed in our study, and led to an inaccurate statement. This correction does not affect the main conclusions and interpretations of the paper.

In the Introduction section, first column, third paragraph, 11th line on page 796, the sentence should be corrected as follows: "Retrograde tracing anatomical results from both the RPa (Hosoya et al., 1987; Hermann et al., 1997; Sarkar et al., 2007) and PaMP (Sawchenko, 1991; Sawchenko et al., 2000; Herman et al., 2003) were reported previously and a recent study has assessed the extent to which RPa and PaMP afferent inputs overlap and display stress-evoked activity (Kataoka et al., 2014).”

The full reference for the added report is:

\section{Reference}

Kataoka, N., Hioki, H., Kaneko, T., Nakamura, K., (2014) Psychological stress activates a dorsomedial hypothalamus-medullary raphe circuit driving brown adipose tissue thermogenesis and hyperthermia. Cell Metab 20:346-358.

DOI: 10.1523/JNEUROSCI.1273-16.2016

\section{Correction: Morillon et al., Temporal Prediction in lieu of Periodic Stimulation}

In the article "Temporal Prediction in lieu of Periodic Stimulation" by Benjaminn Morillon, Charles E. Schroeder, Valentin Wyart, and Luc H. Arnal, which appeared on pages 2342-2347 of the February 24, 2016 issue, the authors regret that not all of the funding sources were cited in the accepted version of the acknowledgments. The corrected acknowledgments are as follows:

“This work was supported by a fellowship from the Montreal Neurological Institute (B.M.), and by the National Institute of Mental Health-National Institutes of Health (Grant MH103814 to C.E.S.). We thank Sylvain Baillet and David Poeppel for support, Peter Lakatos for discussions, and the participants for their willingness to be part of the study."

DOI: 10.1523/JNEUROSCI.1277-16.2016 